\title{
The Heisenberg limit for laser coherence
}

\author{
Howard M. Wiseman*, S. Nariman Saadatmand, Travis J. Baker \\ Centre for Quantum Dynamics, Griffith University, Nathan, Brisbane, QLD 4111, Australia \\ *h.wiseman@griffith.edu.au \\ Dominic W. Berry \\ Department of Physics and Astronomy, Macquarie University, Sydney, NSW 2109, Australia
}

\begin{abstract}
Let $\mathfrak{C}$ be the number of photons in a coherence time. The standard (SchawlowTownes) result is $\mathfrak{C}=O\left(\mu^{2}\right)$, with $\mu$ the laser cavity photon number. Here we establish the Heisenberg limit: $\mathfrak{C}=O\left(\mu^{4}\right)$. (C) 2019 The Author(s)
\end{abstract}

OCIS codes: $030.1640,270.2500,270.3430$.

\section{Introduction}

Quantum theory underpins much modern technological development, and sets the ultimate limits to the performance of devices - the best conceivable performance towards which scientists and engineers can work under the constraint of a given resource (such as energy or power). In this context, a quantum enhancement exists when the ultimate limit, also known as a Heisenberg limit, scales better in terms of the resource than the standard quantum limit (SQL) [1]. The latter is also derived employing quantum theory, but using a set of 'standard' assumptions on how the device must work. The quadratic quantum enhancement found in static phase estimation [2,3] is well known, and there are many other metrological examples [1,4]. Here, by contrast, we prove that there can be a quadratic quantum enhancement in the production of a physical property of great importance for both classical and quantum technology: optical coherence.

A laser beam epitomises optical coherence in all its aspects, including a long coherence time. This time can be converted to a dimensionless measure of coherence, $\mathfrak{C}$, by multiplying by $\mathscr{N}$, the number of photons emitted per unit time. This gives, loosely speaking, the number of photons emitted consecutively that are mutually coherent. The quantum limit to the coherence time was famously studied by Schawlow and Townes over 60 years ago [5]. However, even more rigorous subsequent work [6] made assumptions not entailed by fundamental requirements such as local conservation of energy. In the 21 st century, our ability to engineer and control quantum systems $[7,8]$ has changed our conception of what is practical. At the same time, our understanding of quantum processes has been deepened through theoretical and numerical quantum-information-theory techniques such as operational super-selection rules (SSRs) [9, 10], and matrix product states (MPSs) [11-13]. Hence it is plausible that the Schawlow-Townes limit, $\mathfrak{C}_{\mathrm{SQL}}^{\text {ideal }}=O\left(\mu^{2}\right)$, to laser coherence is only a SQL, and that a Heisenberg limit can be proven to lie beyond it.

The central result of this paper is that the true ultimate limit, the Heisenberg limit, for a laser beam having properties akin to those of the ideal models, is $\mathfrak{C}_{\mathrm{HL}}^{\mathrm{rideal}}=\Theta\left(\mu^{4}\right)$. This quadratic improvement implies vastly better performance in the limit $\mu \gg 1$ which characterises most lasers. We prove the existence of a quadratic quantum enhancement in laser coherence creation by the following steps. First, we state our conditions on the laser and its beam. Second, we show analytically from these the upper bound of $\mathfrak{C}=O\left(\mu^{4}\right)$. Third, guided by our tensor-network description of such an ideal laser, we introduce a $\mu$-parametrized family of laser models and show numerically that $\mathfrak{C}=\Theta\left(\mu^{4}\right)$. Fourth, we relay on some theoretical and numerical results to show that all conditions are satisfied in our tensor-network model.

\section{Conditions defining an ideal laser}

We formally consider the following conditions as defining an ideal laser assumptions, which are sufficient for deriving the Heisenberg limit to the coherence.

1. One-dimensional beam. The beam propagates away from the laser in a particular direction, at a constant speed, and has a single transverse mode and a single polarisation. Mathematically, at any time $T \in \mathbb{R}$, the beam is describable by a one-parameter quantum field $\hat{b}(t)$, satisfying $\left[\hat{b}(t), \hat{b}^{\dagger}(s)\right]=\delta(t-s)$, defined for $t \in(-\infty, T]$, such that $\hat{b}(t)$ is independent of $T$. The argument of $\hat{b}(t)$ is the time at which that infinitesimal part of the beam was created by the laser, and $\hat{b}^{\dagger}(t) \hat{b}(t)$ is the operator for photon flux (photons per unit time).

2. Autochthonous phase. The coherence of the beam proceeds only from the laser. That is, a phase shift imposed on the laser state at some time $T$ will lead, in the future, to the same phase shift on the beam emitted 
after time $T$ as well as on the laser state. The phase shift at time $T$ on the laser (which may have been prepared by measurement on the beam generated prior to $T$ ) is described by the unitary transformation $\hat{U}_{\zeta}=\exp \left(-i \zeta \hat{n}_{\mathrm{c}}\right)$. The effect of this, at any time $T^{\prime}>T$, on the state of the cavity plus the beam segment generated in the interval $\left(T, T^{\prime}\right]$, is described by the unitary transformation $\hat{U}_{\zeta}^{\prime}=\exp \left(-i \zeta\left(\hat{n}_{\mathrm{c}}+\hat{n}_{\mathrm{b}}^{\prime}\right)\right)$, where $\hat{n}_{\mathrm{b}}^{\prime}=\int_{T}^{T^{\prime}} \hat{b}^{\dagger}(s) \hat{b}(s) d s$ is the photon number operator for the generated beam segment.

3. Stationarity. The statistics of the laser and beam are invariant under time translation, in the long-time limit. In particular, the mean of $\hat{n}_{\mathrm{c}}$ has a unique long-time limit, $\mu$.

4. Ideal Glauber ${ }^{(1),(2)}$-coherence. The stationary beam is close to an ideal laser beam [6, 14-16] an eigenstate of $\hat{b}(t)$ of eigenvalue $\beta(t)=\sqrt{\mathscr{N}} e^{i \sqrt{\ell} W(t)}$, with $W(t)$ a Weiner process [16] - in the sense that the beam's first- and second-order Glauber coherence functions [17] well approximate those of the ideal beam. The first- and second-order Glauber coherence functions are defined as $G^{(1)}(s, t)=$ $\left\langle\hat{b}^{\dagger}(s) \hat{b}(t)\right\rangle, G^{(2)}\left(s, s^{\prime}, t^{\prime}, t\right)=\left\langle\hat{b}^{\dagger}(s) \hat{b}^{\dagger}\left(s^{\prime}\right) \hat{b}\left(t^{\prime}\right) \hat{b}(t)\right\rangle . G^{(1)}$ yields the photon flux $\mathscr{N}=G^{(1)}(t, t)$, and the coherence $\mathfrak{C}=\max _{\omega}\left|\int_{-\infty}^{\infty} G^{(1)}(s, t) e^{-i \omega s} d s\right|$. The requirement on $G^{(1)}$ in this Condition is that the laser power spectrum is Lorentzian (or close to it) with linewidth $\ell \equiv 4 \mathscr{N} / \mathfrak{C}$, while the requirement on $G^{(2)}$ implies that the photon statistics are Poissonian (or close to it).

\section{Analytical derivation}

Here, we only sketch the analytical proof of that the above Conditions lead to the upper bound $\mathfrak{C}=O\left(\mu^{4}\right)$. Consider a heterodyne measurement [18] of the laser beam in the interval $[t-\tau, t)$, where $\tau=\sqrt{6 /(5 \mathscr{N} \ell)}$ (this value is chosen to give the tightest bound, below). From the result, the observer can form an estimate, $\phi_{\mathrm{F}}$, of the phase of the laser beam at that time. Consider two methods by which a second, newly arrived, observer can estimate $\phi_{\mathrm{F}}$. The first method is by direct measurement on the laser cavity at time $t$. Now, Condition 2 ensures that information about $\phi_{\mathrm{F}}$ can only be encoded in that quantity conjugate to $\hat{n}_{\mathrm{c}}$, which has mean $\mu$ from Condition 3 . Adapting a result of Ref. [19], the mean sine-squared error (MSSE) of any such estimate is bounded below by $k / \mu^{2}$, where $k \approx 1.8936$. The second method is by heterodyne measurement of the laser beam in the interval $(t, t+\tau]$. From Condition 4 we can show that the MSSE for this measurement is bounded above by $8 \sqrt{10 /\left(3 \mathfrak{C}^{2}\right)}$. Now Condition 2 also implies that the second method accesses only that phase information stored in the cavity at time $t$. Thus the second MSSE cannot be smaller than the first, which cannot be smaller than $k / \mu^{2}$. Hence,

$$
\mathfrak{C}^{\text {ideal }} \lesssim 59.51 \mu^{4}
$$

\section{The tensor network description}

MPS methods are widely used in quantum information theory and condensed matter physics [13, 20], and have had some applications in quantum optics [21,22], but have never been used to describe a laser beam, to the best of our knowledge. In particular, while Ref. [21] points out that a cavity quantum-electrodynamics system can be generally emulated using such a sequential process, the authors were not concerned with describing coherence generation (in the sense defined above) by a laser.

Fig. 1 illustrates our MPS description of an ideal laser system. As pictured in the figure, we are interested in the one-site unit-cell infinite MPS (iMPS) that $\hat{V}$ eventually creates for arbitrary consecutive times $q_{0} \delta t$ and $\left(q_{0}+1\right) \delta$, where $\delta t$ is an arbitrarily small time (connecting the discretized system to the continuum model). In terms of the $A$ operators used above, the iMPS is given by

$$
\left|\Psi_{\mathrm{iMPS}}\right\rangle=\sum_{\ldots, j_{q_{0}}, j_{q_{0}-1}, \ldots}\left\langle\left.\Phi(q=+\infty)\right|_{\mathrm{c}} \cdots A_{\left(q_{0}\right)}^{\left[j_{q_{0}}\right]} \times A_{\left(q_{0}-1\right)}^{\left[j_{q_{0}-1}\right]} \cdots \mid \Phi(q=-\infty)\right\rangle_{\mathrm{c}}\left|\ldots, j_{q_{0}}, j_{q_{0}-1}, \ldots\right\rangle_{\mathrm{o}}
$$

where $|\Phi(q)\rangle_{\mathrm{c}}$ denotes the state of the cavity at integer time $q$. Since there exists translational invariance in the outputs, we now drop the $(q)$-subscripts. This is equivalent to the tensor-network algorithm introduced in Ref. [23] since we assume a fixed-point for the reduced density matrix of the laser cavity, $\rho^{\mathrm{ss}}$, i.e. $\sum_{j} \hat{A}^{[j]} \rho^{\mathrm{ss}} \hat{A}^{[j] \dagger}=\rho^{\mathrm{ss}}$.

To obtain a continuum limit for the beam we set $A^{[2]}=0$, and define two infinitesimal $A$-matrices in terms of a one-photon gain operator $\hat{G}=A^{[0]} / \sqrt{\mathscr{N} \delta t}$ and one-photon loss operator $\hat{L}=A^{[3]} / \sqrt{\mathscr{N} \delta t}$. We do not assume linear damping $\left(L_{n} \propto \sqrt{n}\right)$. Rather, guided by iMPS optimization of $\mathfrak{C}$, we define our family of models by the choices $\rho_{n} \propto \sin ^{4}\left(\pi \frac{n+1}{D+1}\right), G_{n}=1, L_{n} \in \mathbb{R}_{+}$, for which $\mu$ (Condition 3) equals $(D-1) / 2$ and $\mathscr{N}$ (Condition 4) equals 1 (which simply sets a convenient time unit). For our model, the coherence can be evaluated as $\mathfrak{C}=$ $\int_{-\infty}^{\infty} G^{(1)}(s, t) d s=2 \operatorname{Tr}\left[\hat{L}^{\dagger} \mathscr{L}_{+}^{-1}\left(\hat{L} \rho_{\mathrm{ss}}\right)\right]$. We evaluate this for the above family of models, with $D$ up to 1000 and find $\mathfrak{C} \sim 0.0619(8) \mu^{4}$. Moreover, we can show analytically that our model exactly satisfies Conditions $1-3$, and we also showed numerically (not presented here) that Condition 4 is satisfied by our model for $\mu \gg 1$. Hence the scaling of the upper bound in our theorem is achievable, albeit with a far smaller coefficient than that in Eq. (1). 

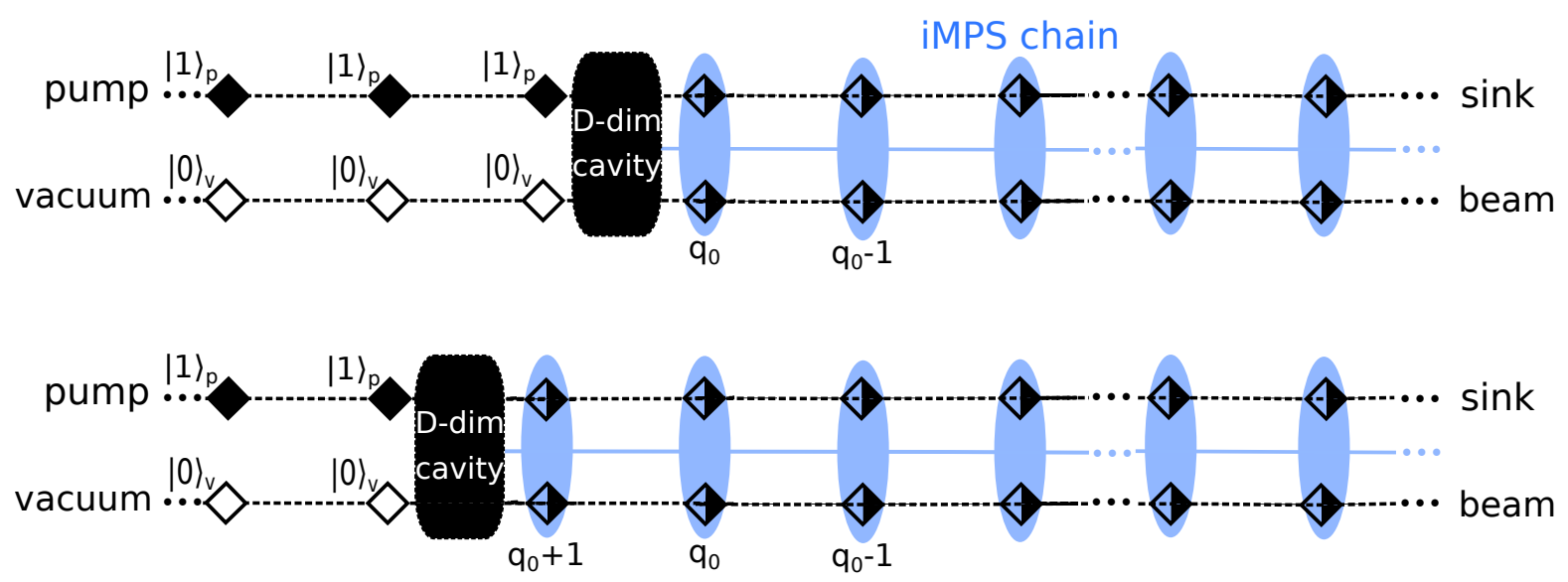

Fig. 1. Conceptual diagram of our iMPS laser model containing five elements: the cavity (c), a pump, a vacuum input, the beam output (b), and a sink (s), all of which are essential for laser operation. We consider the sink and beam as a joint four-level system. The time evolution of the cavity (c) is governed by the generative interaction $\hat{V}_{q}=\sum_{j_{q+1}, m, n} A_{m n}^{\left[j_{q+1}\right]}\left|j_{q+1}\right\rangle_{\mathrm{o}}|m\rangle_{\mathrm{c}}\langle n|$, which maps a $D$-dimensional vector space into a $4 \times D$-dimensional one.

\section{Conclusion}

The Schawlow-Townes limit to laser coherence $\mathfrak{C}$ (the number of mutually coherent photons emitted in the beam) is only a standard quantum limit. Beyond it lies a Heisenberg limit, quadratically better in terms of $\mu$ (the number of excitations in the laser itself). For $\mu$ large this represents a vast improvement in laser coherence properties. We constructed a model that achieves this quantum enhancement in scaling, while retaining the same first- and secondorder coherence properties as an ideal laser beam (a constant-intensity coherent state with a diffusing phase). The assumption of these coherence properties is necessary to prove the Heisenberg limit $\mathfrak{C}_{\mathrm{HL}}^{\mathrm{ideal}}=O\left(\mu^{4}\right)$.

\section{References}

1. V. Giovannetti, S. Lloyd, L. Maccone, Science 306, 1330 (2004).

2. C. M. Caves, Phys. Rev. D 23, 1693 (1981).

3. B. Yurke, S. L. McCall, J. R. Klauder, Phys. Rev. A 33, 4033 (1986).

4. D. Berry, M. Hall, H. Wiseman, Phys. Rev. Lett. 111, 113601 (2013).

5. A. L. Schawlow, C. H. Townes, Phys. Rev. 112, 1940 (1958).

6. H. M. Wiseman, Phys. Rev. A 60, 4083 (1999).

7. C. Sayrin, et al., Nature 477, 73 (2011).

8. K. S. Chou, et al., Nature 561, 368 (2018).

9. S. D. Bartlett, H. M. Wiseman, Phys. Rev. Lett. 91, 097903 (2003).

10. S. D. Bartlett, T. Rudolph, R. W. Spekkens, Rev. Mod. Phys. 79, 555 (2007).

11. I. Affleck, T. Kennedy, E. H. Lieb, H. Tasaki, Phys. Rev. Lett. 59, 799 (1987).

12. D. Perez-Garcia, F. Verstraete, M. M. Wolf, J. I. Cirac, Quantum Info. Comput. 7, 401 (2007).

13. U. Schollwock, Annals of Physics 326, 96 (2011). January 2011 Special Issue.

14. W. H. Louisell, Quantum Statistical Properties of Radiation (John Wiley \& Sons, New York, 1973).

15. M. Sargent, M. O. Scully, W. E. Lamb, eds., Laser Physics (Addison-Wesley, Reading Mass., 1974).

16. H. Carmichael, Statistical Methods in Quantum Optics 1 (Springer, 1999).

17. R. J. Glauber, Phys. Rev. 130, 2529 (1963).

18. H. M. Wiseman, G. J. Milburn, Quantum Measurement and Control (Cambridge University Press, Cambridge, England, 2010).

19. A. Bandilla, H. Paul, H. H. Ritze, Quantum Optics: Journal of the European Optical Society Part B 3, 267 (1991).

20. R. Orús, Annals of Physics 349, 117 (2014).

21. C. Schön, E. Solano, F. Verstraete, J. I. Cirac, M. M. Wolf, Phys. Rev. Lett. 95, 110503 (2005).

22. M. Jarzyna, R. Demkowicz-Dobrzanski, Phys. Rev. Lett. 110, 400 (2013).

23. I. P. McCulloch, ArXiv e-prints 0804.2509 (2008). 